\title{
Comparison of CBF Measured with Combined Velocity- Selective Arterial Spin-Labeling and Pulsed Arterial Spin- Labeling to Blood Flow Patterns Assessed by Conventional Angiography in Pediatric Moyamoya
}

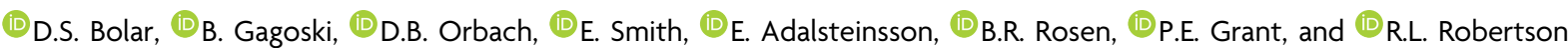

\begin{abstract}
BACKGROUND AND PURPOSE: Imaging CBF is important for managing pediatric moyamoya. Traditional arterial spin-labeling MR imaging detects delayed transit thorough diseased arteries but is inaccurate for measuring perfusion because of these delays. Velocity-selective arterial spin-labeling is insensitive to transit delay and well-suited for imaging Moyamoya perfusion. This study assesses the accuracy of a combined velocity-selective arterial spin-labeling and traditional pulsed arterial spin-labeling CBF approach in pediatric moyamoya, with comparison to blood flow patterns on conventional angiography.
\end{abstract}

MATERIALS AND METHODS: Twenty-two neurologically stable pediatric patients with moyamoya and 5 asymptomatic siblings without frank moyamoya were imaged with velocity-selective arterial spin-labeling, pulsed arterial spin-labeling, and DSA (patients). Qualitative comparison was performed, followed by a systematic comparison using ASPECTS-based scoring. Quantitative pulsed arterial spin-labeling CBF and velocity-selective arterial spin-labeling CBF for the middle cerebral artery, anterior cerebral artery, and posterior cerebral artery territories were also compared.

RESULTS: Qualitatively, velocity-selective arterial spin-labeling perfusion maps reflect the DSA parenchymal phase, regardless of postinjection timing. Conversely, pulsed arterial spin-labeling maps reflect the DSA appearance at postinjection times closer to the arterial spin-labeling postlabeling delay, regardless of vascular phase. ASPECTS comparison showed excellent agreement $(88 \%, \kappa=0.77$, $P<.001)$ between arterial spin-labeling and DSA, suggesting velocity-selective arterial spin-labeling and pulsed arterial spin-labeling capture key perfusion and transit delay information, respectively. CBF coefficient of variation, a marker of perfusion variability, was similar for velocity-selective arterial spin-labeling in patient regions of delayed-but-preserved perfusion compared to healthy asymptomatic sibling regions (coefficient of variation $=0.30$ versus 0.26 , respectively, $\Delta$ coefficient of variation $=0.04$ ), but it was signifcantly different for pulsed arterial spin-labeling (coefficient of variation $=0.64$ versus $0.34, \Delta$ coefficient of variation $=0.30$, $P<.001)$.

CONCLUSIONS: Velocity-selective arterial spin-labeling offers a powerful approach to image perfusion in pediatric moyamoya due to transit delay insensitivity. Coupled with pulsed arterial spin-labeling for transit delay information, a volumetric MR imaging approach capturing key DSA information is introduced.

ABBREVIATIONS: $\mathrm{ACA}=$ anterior cerebral artery; $\mathrm{ASL}=$ arterial spin-labeling; $\mathrm{CV}=$ coefficient of variation; PASL $=$ pulsed arterial spin-labeling; PCA = posterior cerebral artery; PCASL = pseudocontinuous arterial spin-labeling; PLD = postlabeling delay; VSASL = velocity-selective arterial spin-labeling

erfusion imaging plays an important role in the management of patients with moyamoya. Conventional catheter DSA is
Received April 24, 2019; accepted after revision August 21.

From the Department of Radiology (D.S.B.) and Center for Functional Magnetic Resonance Imaging (D.S.B.), UC San Diego, La Jolla, California; Fetal Neonatal Neuroimaging and Developmental Science Center (B.G., P.E.G.), Department of Radiology (B.G., D.B.O., P.E.G., R.L.R.), Division of Neurointerventional Radiology (D.B.O.), Department of Neurosurgery (E.S.), and Division of Newborn Medicine (P.E.G.), Department of Medicine, Boston Children's Hospital, Boston,

Massachusetts; Department of Electrical Engineering \& Computer Science (E.A.),

Massachusetts Institute of Technology, Cambridge, Massachusetts; and MGH/HST

Athinoula A. Martinos Center for Biomedical Imaging (E.A., B.R.R.), Charlestown, Massachusetts. the criterion standard technique used to assess intracranial arteriopathy and blood flow patterns, but it is invasive, uses ionizing radiation, and introduces a small risk of neurologic

Paper previously presented, in part, at: Annual Meeting of the International Society for Magnetic Resonance in Medicine, May 30 to June 5, 2015; Toronto, Ontario, Canada; and Annual Meeting of the Radiological Society of North America, November 29 to December 4, 2015; Chicago, Illinois.

Please address correspondence to Divya S. Bolar, MD, PhD, UCSD Center for Functional Magnetic Resonance Imaging, W.M. Keck Building, 9500 Gilman, 0677, La Jolla, CA 92037-0677; e-mail: dbolar@ucsd.edu

Indicates article with supplemental on-line photos.

http://dx.doi.org/10.3174/ajnr.A6262 


\begin{tabular}{|c|c|c|c|c|c|c|c|c|}
\hline Pt. & $\begin{array}{c}\text { Moyamoya } \\
\text { Etiology }\end{array}$ & $\begin{array}{l}\text { Age } \\
(y r)\end{array}$ & Sex & Laterality & $\begin{array}{c}\text { Operative } \\
\text { Status }\end{array}$ & $\begin{array}{c}\text { Suzuki }^{37} \text { (R/L), } \\
\text { Preoperative }\end{array}$ & Treatment & $\begin{array}{c}\text { Matsushima }^{38} \\
\text { (R/L) }\end{array}$ \\
\hline \multirow[t]{2}{*}{1} & Idiopathic & 11 & $\mathrm{~F}$ & Left & Pre & IV & & \\
\hline & & 12 & & & Post & & Left synangiosis & C \\
\hline 2 & NFI & 6 & M & Right & Post & IV-V & Right synangiosis & A \\
\hline 3 & $S C D$ & 13 & $\mathrm{~F}$ & Bilateral & Post & NA & Bil synangiosis & A \\
\hline 4 & Idiopathic & 14 & $\mathrm{~F}$ & Bilateral & Post & III-IV/III-IV & Bil synangiosis & $\mathrm{A} / \mathrm{A}$ \\
\hline 5 & Idiopathic & 19 & $\mathrm{~F}$ & Bilateral & Post & V-VI/III-IV & Bil synangiosis & $\mathrm{A} / \mathrm{C}$ \\
\hline 6 & $\mathrm{NFI}$ & 17 & $\mathrm{~F}$ & Bilateral & Pre & I/III-IV & & \\
\hline 7 & SCD & 11 & M & Bilateral & Post & II/II & Bil synangiosis & $A / B$ \\
\hline 8 & Idiopathic & 14 & M & Bilateral & Post & IV/IV & Bil synangiosis & $\mathrm{A} / \mathrm{A}$ \\
\hline 9 & Idiopathic & 13 & $\mathrm{~F}$ & Bilateral & Post & II/III & Bil synangiosis & $\mathrm{B} / \mathrm{C}$ \\
\hline 10 & Idiopathic & 16 & $\mathrm{~F}$ & Bilateral & Pre & $\mathrm{I} / \mathrm{I}$ & & \\
\hline 11 & Idiopathic & 7 & $\mathrm{~F}$ & Bilateral & Pre & $\mathrm{II} / \mathrm{II}$ & & \\
\hline 12 & $\mathrm{NFF}^{\mathrm{a}}$ & 5 & $\mathrm{~F}$ & Right & Pre & 1 & & \\
\hline 13 & SCD & 11 & $\mathrm{~F}$ & Right & Pre & ॥ & & \\
\hline 14 & NFI & 8 & M & Left & Post & III & Left synangiosis & A \\
\hline 15 & Idiopathic & 7 & $\mathrm{~F}$ & Right & Post & III & Right synangiosis & A \\
\hline 16 & Idiopathic & 13 & $\mathrm{~F}$ & Left & Post & NA & Left synangiosis & B \\
\hline 17 & Idiopathic & 19 & $\mathrm{~F}$ & Bilateral & Pre & III/I-II & & \\
\hline 18 & Idiopathic & 3 & M & Bilateral & Post & II-III/II-III & Bil synangiosis & $B / A-B$ \\
\hline 19 & NFI & 15 & M & Left & Post & V & Left synangiosis & B \\
\hline 20 & Idiopathic & 12 & M & Right & Post & III-IV & Right synangiosis & $B-C$ \\
\hline 21 & Idiopathic & 15 & M & Bilateral & Post & III/III & Bil synangiosis & $B / B$ \\
\hline 22 & Idiopathic & 2 & M & Bilateral & Post & IV/II & Bil synangiosis & $A / A-B$ \\
\hline
\end{tabular}

Note:-NF1 indicates neurofibromatosis type 1; SCD, sickle cell disease; Pre, before; Post, after; R, right; L, left; NA, not applicable; Bil, bilateral.

a Patient with NFI with radiated right masticator space tumor; arteriopathy possibly related to radiation.

complication. ${ }^{1,2}$ Therefore, noninvasive imaging techniques remain attractive to reduce the necessity of DSA for evaluation of cerebral blood flow.

Standard MR perfusion approaches such as pulsed and pseudocontinuous arterial spin-labeling (PASL and pCASL) are limited in imaging moyamoya perfusion because they are affected by prolonged arterial arrival times secondary to the arteriopathy. Arrival of the arterial spin-labeling (ASL) magnetic label to the brain parenchyma is subsequently delayed, resulting in macrovascular signal (so-called arterial transit artifact) and artifactual areas of perfusion deficit. ${ }^{3,4}$

Velocity-selective ASL (VSASL) is a newer perfusion technique that relies on imaging principles not affected by variations in arterial arrival time. Using velocity-selective labeling modules, VSASL creates the magnetic label in small arteries within the imaging voxels themselves, allowing immediate delivery to target microvasculature, circumventing transit delay errors. ${ }^{5}$ A recent study compares VSASL, pCASL, and xenon-enhanced CT CBF in adult patients with moyamoya ${ }^{6}$ and supports these hypotheses but lacks comparison with DSA. Furthermore, xenon is not FDA-approved for pediatric use. ${ }^{7}$

The current study evaluates a combined VSASL and PASL protocol in pediatric moyamoya and adds a critical comparison with blood flow patterns assessed by DSA. We hypothesized the following: 1) VSASL is insensitive to arterial transit delays, allowing assessment of parenchymal perfusion; and 2) PASL is sensitive to arterial transit delays, facilitating identification of affected territories, but it is inaccurate for measuring perfusion within these territories. We believe that each ASL approach provides unique but complementary information about cerebral hemodynamics in moyamoya. To investigate these hypotheses, we performed a systematic comparison of PASL/VSASL to DSA and compared perfusion variability measured with VSASL and PASL in disease-affected regions.

\section{MATERIALS AND METHODS \\ Subjects}

The study was approved by the Boston Children's Hospital institutional review board; informed consent was not required. PASL and VSASL sequences were added to moyamoya MR imaging protocols in late 2014 after a short trial period, resulting in approximately 100 pediatric patients with moyamoya and 5 asymptomatic siblings scanned during 3 years. Of these, 23 patients were selected on the basis of the following inclusion criteria: stable appearance of stenoses and collaterals between the time of MRI/MRA and DSA (either pre- or postsynangiosis), closely matched slice position between VSASL and PASL, and lack of recent neurologic events. For studies $>2$ months apart, vascular stability was confirmed by comparing the MRA appearance before and after the DSA (in addition to direct comparison to DSA itself). Using these criteria, 22 patients were selected, after excluding 1 patient due to ASL artifacts (primarily related to motion and susceptibility from surgical hardware). For one of the patients, both preoperative and postoperative data were included. Data from 5 asymptomatic sibling screens thought not to have frank moyamoya were also included and consisted of ASL data only, except for 1 sibling who also underwent conventional angiography due to initially suspected disease by MRA. Subjects ranged from 2 to 19 years of age, 17 female and 9 male (patients listed in Table 1).

\section{Data Acquisition}

MRI/MRA scans were performed at 3T (Siemens Trio and Skyra, Erlangen, Germany) and included PASL, VSASL, and standard anatomic/angiographic sequences.

AJNR Am J Neuroradiol 40:1842-49 Nov 2019 www.ajnr.org 


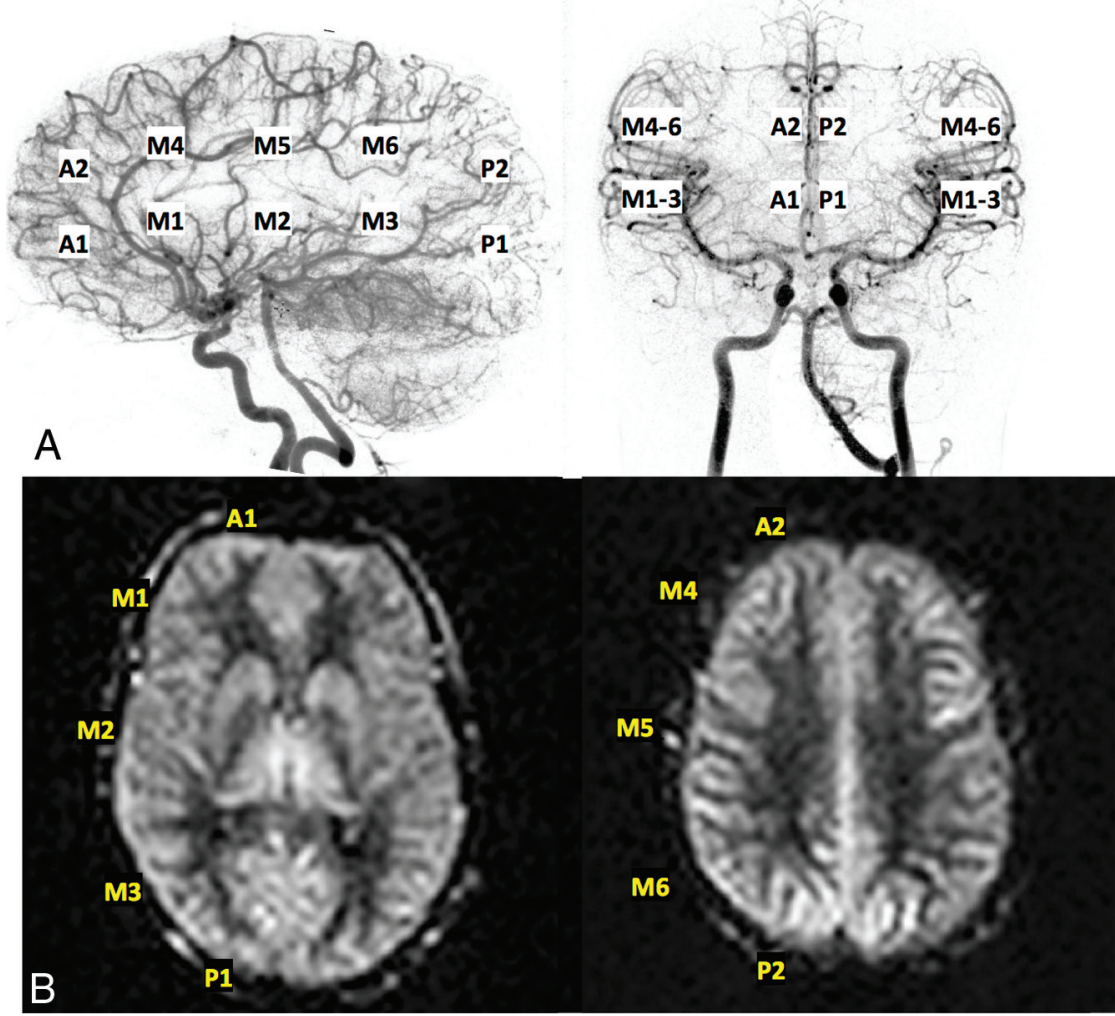

FIG 1. Representative ASPECTS regions used for scoring of both DSA (A) and ASL data (B); specifcally, 2 ACA regions, 6 MCA regions, and 2 posterior cerebral artery regions per side were evaluated.

VSASL-specific parameters were $\mathrm{V}_{\text {CUTOFF }}=2.1 \mathrm{~cm} / \mathrm{s}$ and TI/ postlabeling delay $(\mathrm{PLD})=1300 \mathrm{~ms}$ to the center slice (VSASLPLD). PASL (quantitative imaging of perfusion using a single subtraction II [QUIPSS II] with thin-slice saturation) specific parameters were $\mathrm{TI}_{1}=700 \mathrm{~ms}, \mathrm{TI}_{1, \text { stop }}=1400 \mathrm{~ms}$, and $\mathrm{TI} /$ $\mathrm{PLD}=2000 \mathrm{~ms}$ to the center slice (PASL-PLD), and PASL label width $=110 \mathrm{~mm}$. VSASL parameters are similar to those used in initial studies previously found to target parenchymal perfusion, 5,8 and PASL parameters were adapted on the basis of ASL white paper recommendations. ${ }^{9}$ The imaging module for both PASL and VSASL included an EPI readout with TE/TR $=13 / 3000 \mathrm{~ms}$, voxel size $=3.5 \times 3.5 \times 5.0 \mathrm{~mm}^{3}, 20$ slices, bandwidth $=2300 \mathrm{~Hz} /$ pixel, 90 measurements, and scan time $=4$ minutes 30 seconds. A single EPI scan $(<1$ second) was acquired for absolute CBF quantification, the so-called M0 scan. ${ }^{10}$

DSA was performed using a biplane cerebral angiographic system (Axiom Artis dBA Twin; Siemens). Images of ICA, external carotid artery, and vertebral artery injections were obtained in lateral and frontal projections. Temporal resolution for DSA was typically 2 frames/s. Postinjection time is defined as the time between the frame in which contrast first appears and the frame of interest.

\section{Data Analysis}

Data processing for PASL and VSASL was identical except for the exact equations used for quantification. Generation of absolute CBF maps involved an analysis stream based on standard ASL approaches and equations described in detail elsewhere., ${ }^{5,11}$
PASL-CBF and VSASL-CBF maps were qualitatively compared to each other noting discrepant parenchymal signal and areas of PASL macrovascular artifacts. Coronal and sagittal multiplanar reformats of ASL perfusion maps were generated to facilitate comparison to frontal and lateral DSA projections. Attention was paid to whether the ASL-CBF maps resembled the DSA parenchymal phase or arterial phase.

Months later, a more rigorous, systematic comparison between ASL and DSA was performed by 2 independent graders, respectively, using a modified ASPECTS scoring system. ${ }^{4,12}$ DSA and PASL/VSASL data were graded using a 4-point system that evaluates regions corresponding to 10 ASPECTS locations (2 anterior cerebral artery [ACA], $6 \mathrm{MCA}$, and 2 posterior cerebral artery [PCA]) over 2 standard ASPECTS levels (Fig 1). The DSA scale is the following: 0 , absence of capillary blush; 1 , incomplete capillary blush (ie, decreased parenchymal filling of the ASPECT region); 2, complete-but-delayed capillary blush (eg, due to proximal stenosis and/or collaterals); and 3, complete blush from anterograde flow, without delay. The analogous ASL scale is the following: 0, complete absence of parenchymal perfusion by VSASL; 1, incomplete parenchymal perfusion by VSASL (ie, partially absent or conspicuously decreased perfusion within the region); 2, delayed-but-complete parenchymal perfusion as assessed by PASL and VSASL (ie, VSASL demonstrates complete parenchymal perfusion, but PASL shows arterial transit artifact and/or perfusion deficits); and 3, complete/normal perfusion (ie, both VSASL and PASL show approximately matched, homogeneous parenchymal perfusion, without obvious deficit). On-line Figure 1 shows ASL scoring in further detail. An additional subscore of U could be granted, for "uncertain" regions whose score represents a best guess. DSA was scored by an attending pediatric neuroradiologist and former interventionalist (R.L.R.), and ASL was scored by a senior neuroradiology fellow (D.S.B.). Grading was performed in a randomized fashion, with the reviewers blinded to each other's scores. Agreement between the DSA and ASL scores was assessed using the Cohen $\kappa$ coefficient (Matlab; MathWorks, Natick, Massachusetts).

A quantitative PASL-CBF and VSASL-CBF analysis was also performed on 19 patients and all asymptomatic siblings, but it could not be performed on 3 patients due to misalignment between the perfusion-weighted scan and the M0 calibration scan. CBF maps were normalized to a standard pediatric atlas (Montreal Neurological Institute, Montreal, Canada) ${ }^{13}$ and overlaid with vascular territory masks based on microanatomic studies $^{14,15}$ to allow regional measurements. Mean CBF and SD were 

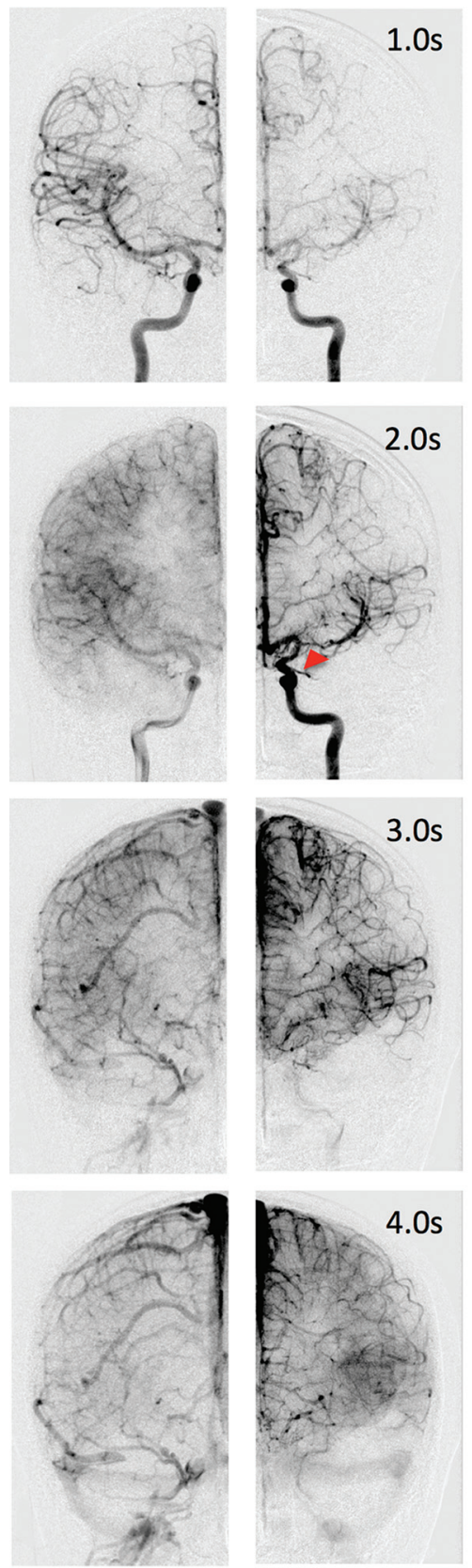

\section{A RICA}

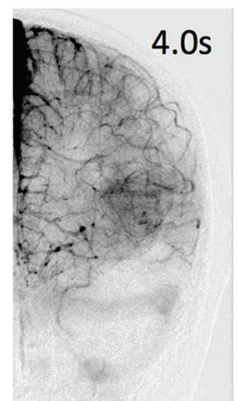

LICA

$.0 \mathrm{~s}$
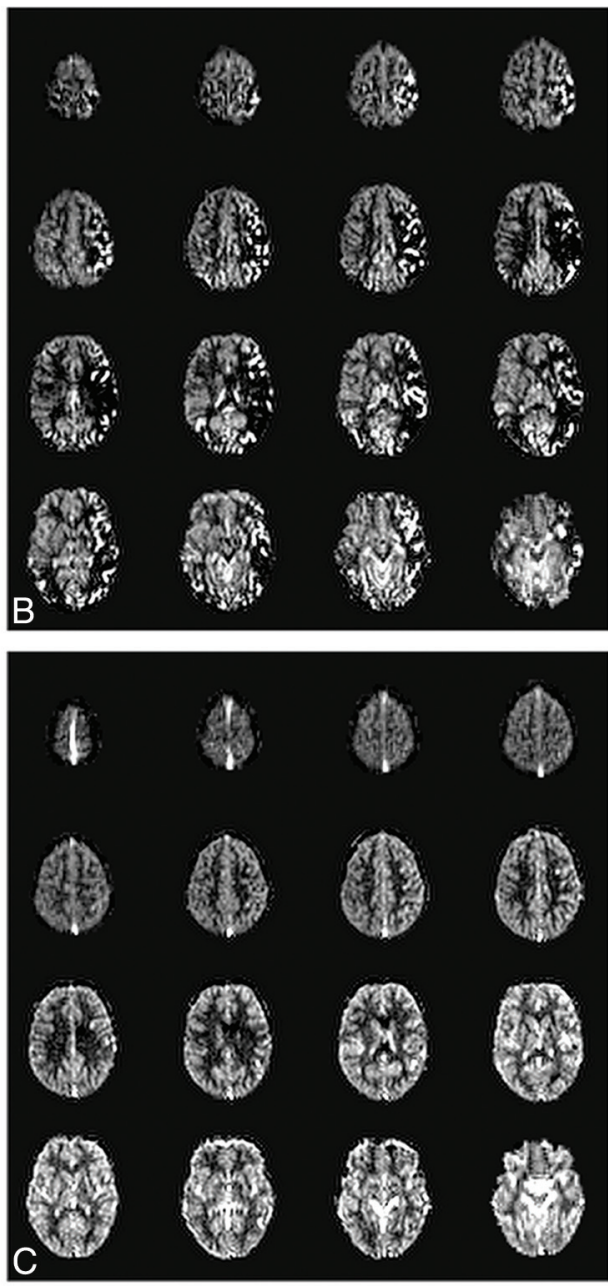

FIG 2. Representative DSA data (A), PASL CBF maps (B), and VSASL CBF maps (C) for a preoperative patient with left-sided moyamoya. A, Frontal DSA data from bilateral ICA injections at various postinjection times. The right side appears relatively normal, with early arterial filling and ACA/MCA parenchymal blush at 2.0 seconds. In contrast, the left side demonstrates delayed anterograde filling through a proximal M1 MCA stenosis (red arrowhead), retrograde filling via ACAMCA collaterals, and delayed parenchymal perfusion of the MCA territory. Parenchymal perfusion of the left MCA territory is finally reached by 4.0 seconds. PASL maps (B) reflect the DSA appearance at 2.0 seconds bilaterally, including areas of curvilinear hyperintensity corresponding to macrovascular flow and perfusion deficit, while VSASL maps (C) reflect parenchymal DSA phases, despite these occurring at different times $(2.0$ seconds on the right, 4.0 seconds on the left). RICA indicates right ICA injection; LICA, left ICA injection.

measured for ACA, MCA, and PCA vascular territories. The coefficient of variation (CV), a marker of perfusion variability, was then calculated by dividing the mean by the SD. Due to frequent macrovascular artifacts seen on PASL-CBF maps, values of
$>115 \mathrm{~mL} / 100 \mathrm{~g} / \mathrm{min}$ were excluded from mean, SD, and CV calculations because this threshold was thought to be above physiologic perfusion based on published pediatric CBF values. ${ }^{16,17}$ PASL and VSASL-CBF were compared in a pair-wise fashion between patients and asymptomatic siblings on a territory-by-territory basis using a Wilcoxon signed rank test (Matlab). Additionally, patient PASL and VSASL CVs of territories with delayed-butcomplete capillary blush by DSA (ie, $\geq 80 \%$ territory with a score of 2 ) were compared with asymptomatic sibling CVs using a Wilcoxon rank-sum test (Matlab).

\section{RESULTS}

\section{Comparisons of DSA, PASL, and VSASL}

For nearly all subjects with DSA and ASL data, PASL appearance is qualitatively similar to DSA appearance at postinjection times close to the PASLPLD, regardless of vascular phase. Conversely, VSASL appearance closely matches the DSA parenchymal phase appearance, regardless of the postinjection time required to reach the parenchymal phase.

Figure 2 shows representative DSA, PASL-CBF, and VSASL-CBF data of a patient with unilateral disease. Frontal DSA from bilateral ICA injections is presented at multiple postinjection times, with excellent correlation to ASL.

On-line Figures 2 and 3 provide additional sets of DSA, PASL, and VSASL data, further highlighting ASL/ DSA correlation. On-line Figure 2 provides data from subject 7 , a patient who underwent bilateral pial synangiosis. On-line Figure 3 provides data from subject 9, a patient post-bilateral pial synangiosis with a chronic right MCA infarction.

\section{ASPECTS Comparisons between DSA and PASL/VSASL}

Table 2 summarizes ASPECTS-based scoring of DSA and ASL data for the 23 subjects with both DSA and ASL data (noting patient 1 with both preoperative and postoperative data). Excellent agreement between DSA and ASL was achieved, with $88 \%$ agreement and observed Cohen $\kappa=0.77, P<.001$. Specifically, 420/480 ASPECTS regions 
had identical scores, and 60/480 regions had discordant scores. U subscores were given to 51 DSA and 15 ASL regions. Notably, 35\% of discordant regions had U subscores (19 DSAs, 2 ASLs), with 15 of these regions graded $1 \mathrm{U}$ on DSA and 2 or 3 on ASL.

\section{Quantitative CBF with PASL and VSASL}

Figure 3 provides a graphical representation of PASL and VSASL average $\mathrm{CBF} C V$ values in patient vascular territories (ie, ACA, MCA, and PCA) affected by transit delay, but ultimately with complete parenchymal perfusion by DSA (44/120), and all asymptomatic sibling vascular territories (30/30). High PASLCBF variability for patients is highlighted (mean CV $=0.64$ compared to 0.34 for asymptomatic sibling scans, statistically different at $P<.001$ ), which is discordant with the homogeneous parenchymal perfusion seen by DSA. Conversely, VSASL-CBF in the same regions demonstrated significantly less variability compared with PASL-CBF (mean VSASL-CBF CV $=0.30$, statistically different from PASL-CBF CV $=0.64$ at $P<.001)$ and is similar to that of asymptomatic sibling scans (VSASL-CBF CV $=0.26$, though still statistically different at $P<.005)$ and concordant with homogeneous parenchymal perfusion on DSA.

Table 2: Contingency table of ASPECTS-based scoring for ASL and DSA data ${ }^{a}$

\begin{tabular}{lccccc}
\hline & \multicolumn{5}{c}{ ASL Score } \\
\cline { 2 - 5 } DSA Score & $\mathbf{0}$ & $\mathbf{1}$ & $\mathbf{2}$ & $\mathbf{3}$ & Totals \\
\hline 0 & 0 & 1 & 0 & 0 & $1(<1 \%)$ \\
1 & 0 & 6 & 17 & 1 & $24(5 \%)$ \\
2 & 0 & 7 & 192 & 21 & $220(46 \%)$ \\
3 & 0 & 0 & 13 & 222 & $235(49 \%)$ \\
Totals & $0(0 \%)$ & $14(3 \%)$ & $222(46 \%)$ & $244(51 \%)$ & 480 \\
\hline
\end{tabular}

${ }^{\mathrm{a}}$ Agreement $=88 \%$, Cohen $\kappa=0.77, P<.0001$.

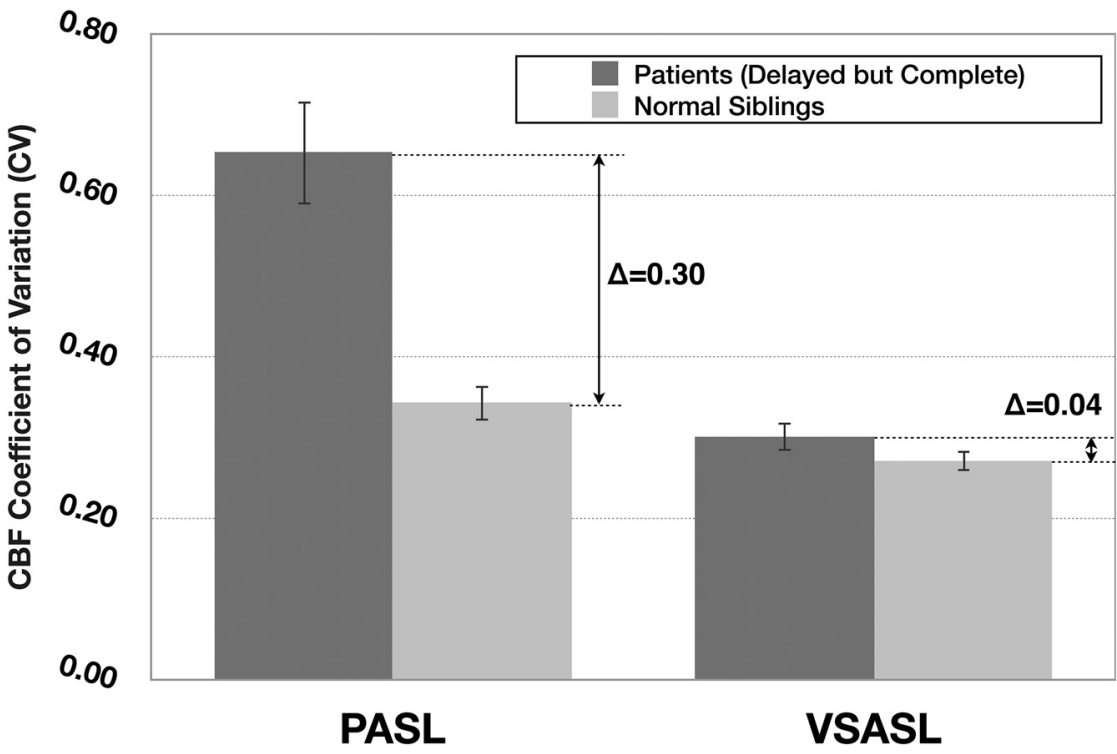

FIG 3. Average PASL and VSASL-CBF CV (a marker of perfusion variability) calculated for patient vascular territories demonstrating delayed-but-complete parenchymal perfusion by DSA, with normal sibling values provided for comparison. Patient VSASL values are similar to those of healthy siblings, consistent with the DSA appearance, but PASL values are markedly different. Error bars denote $95 \% \mathrm{Cl}$.
CBF values themselves were statistically different between VSASL and PASL in patients, when compared on a pair-wise, territory-by-territory basis, yet they were not statistically different in the asymptomatic sibling group (both at $P<.05$ ).

\section{DISCUSSION}

VSASL offers a powerful approach to image perfusion in pediatric moyamoya due its transit delay insensitivity. Traditional PASL and pCASL are limited in moyamoya because they use a spatially defined magnetic label that flows from the proximal arteries and accumulates in distal microvasculature during the PLD, analogous to transit of contrast during DSA (Fig 4). Due to a spatial separation between the arterial label and microvascular imaging regions, a temporal delay arises between labeling and microvascular arrival, the so-called arterial transit delay. ${ }^{10}$ As these the transit delays elongate in moyamoya due to stenoses and collateral pathways, PASL/pCASL techniques become increasingly inaccurate for measuring perfusion. This phenomenon results in the following: 1) visualization of larger arteries proximal to the microvasculature (ie, arterial transit artifact $)^{18,19}$, since the label fails to clear these arteries during the PLD, and 2) absence of microvascular signal and apparent perfusion deficits because the label fails to sufficiently accumulate in the microvasculature by the PLD. ${ }^{4,10,20}$ These effects are depicted in Fig 4B, $-D$. Thus, PASL and pCASL do not always reflect parenchymal perfusion, rendering perfusion assessment in moyamoya by traditional ASL techniques difficult and, in many cases, impossible., ${ }^{4,6,21}$

Conversely, VSASL uses velocity-selective modules to create the magnetic label within small arteries in the imaging slice itself, allowing immediate delivery to the microvasculature during the PLD. VSASL is theoretically insensitive to transit delay and should not experience contamination from macrovascular artifacts or associated apparent perfusion deficits like those seen with PASL in Fig 4D. In situations without pronounced transit delays, VSASL performs comparably with PASL and can similarly identify regions of altered cerebral perfusion. ${ }^{22}$

Our results show that in moyamoya, 1) VSASL is largely insensitive to arterial transit delays, allowing accurate assessment of parenchymal perfusion, and 2) PASL is highly sensitive to arterial transit delays, facilitating identification of affected territories, but inaccurate for measuring perfusion within these territories. These assessments were made by comparing ASL data with the temporal and spatial characteristics of the intracranial circulation with DSA (whose dynamic acquisition captures both macrovascular and microvascular phases of circulation) and also by comparing ASL perfusion variability (CBF $\mathrm{CV}$ ) in patients and asymptomatic siblings not thought to have frank moyamoya. 


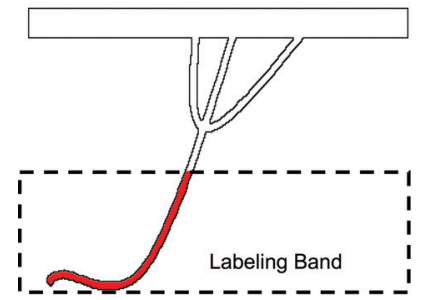

A

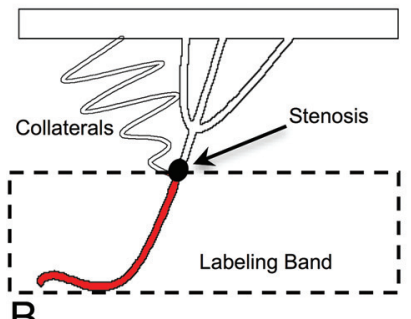

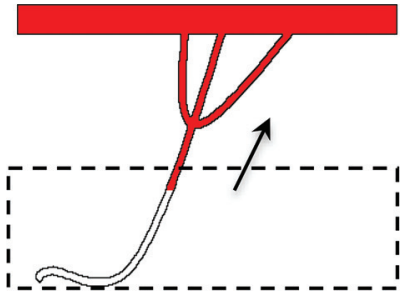

Postlabeling Delay (PLD)
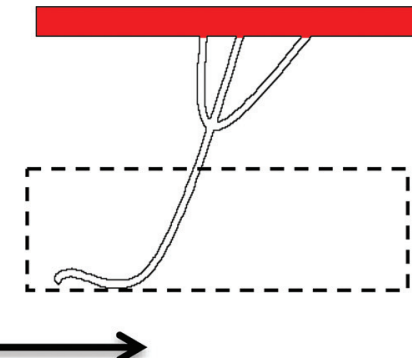
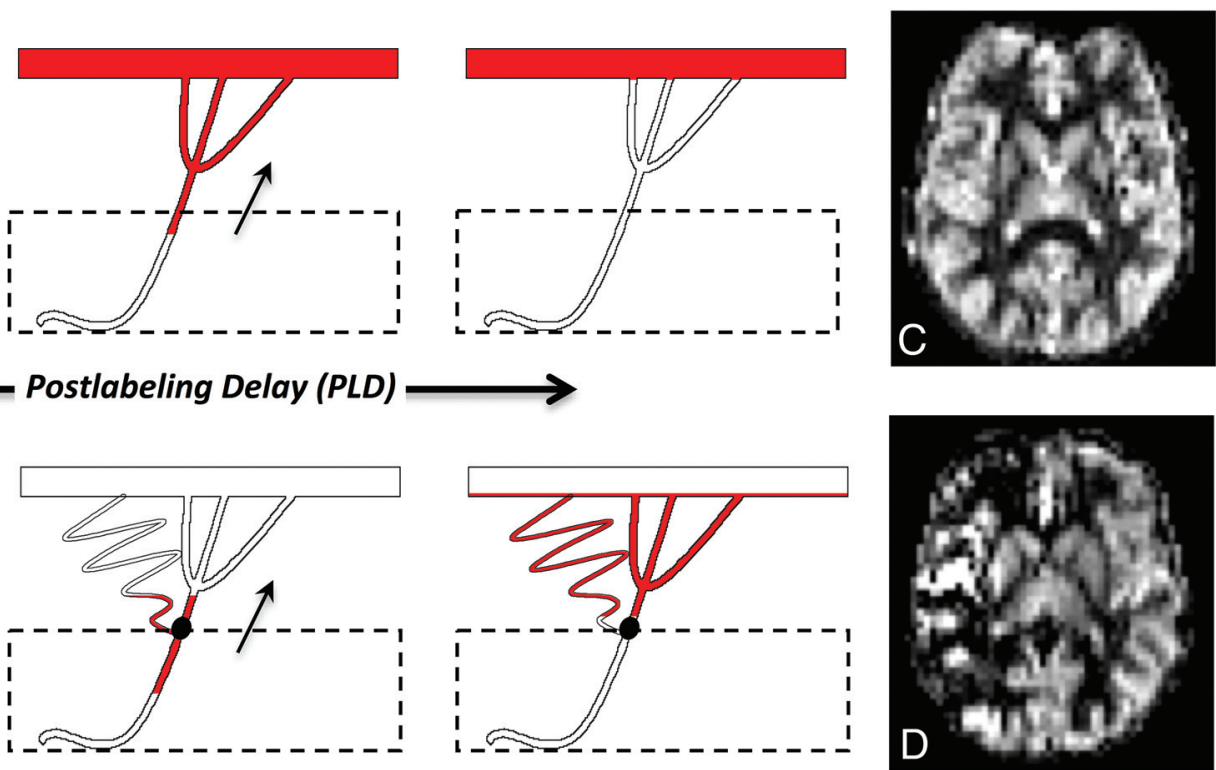

FIG 4. Standard ASL label propagation with patent proximal vessels $(A)$ and steno-occlusive disease with secondary collateralization (B). $A$, The ASL label travels from the labeling band to the distant microvasculature during the standard PLD, resulting in symmetric, homogeneous gray matter perfusion (C). B, The label is delayed due to slow flow through the stenosis and circuitous collateral pathways. Consequently, the label does not fully reach the distal microvasculture during PLD and remains caught in the macrovasculature, resulting in areas of apparent perfusion deficit and hyperintense arterial transit artifacts $(D)$.

Qualitatively, VSASL shows homogeneous and symmetric parenchymal perfusion in cortical gray matter for nearly all patients, consistent with robust compensation and known neurologic and conventional MR imaging stability. These regions consistently corresponded to areas of parenchymal blush seen on DSA, regardless of blush timing. PASL, on the other hand, shows areas of perfusion deficit and hyperintense macrovascular flow, both secondary to delayed arterial transit, corresponding to the DSA appearance at a fixed postinjection time closer to the PASLPLD. It is clear that PASL can result in erroneous perfusion, resulting in perceived perfusion deficits that do not truly exist (best highlighted in Fig 2 and On-line Fig 2). In patients with real perfusion deficits, for example in a patient with a known infarct (On-line Fig 3), PASL transit errors can mask the true perfusion deficit on a background of artifactually deficient regions. In this example, VSASL easily identifies the truly deficient region, discriminating true hypoperfusion from apparent hypoperfusion.

Compared with a purely qualitative analysis, the newly introduced ASPECTS analysis offers a more rigorous and systematic approach for evaluating ASL and DSA data. Excellent agreement between ASL and DSA was observed (88\%, $\kappa=0.77, P<.001)$, which would have been even higher if $U$ regions were excluded. Interestingly, there were nearly 3-4 times as many uncertain regions for DSA $(n=51)$ compared to ASL $(n=15)$. This discrepancy was thought to reflect challenges in localizing perfusion in three dimensions using an inherently two dimensional DSA technique confounded by vessel overlap, cross-filling via contralateral hemispheric branches, and mental summation of multiple single-vessel injections. This limitation of DSA is highlighted by the fact that $79 \%$ of uncertain regions scored as incomplete perfusion by DSA ("1U") were unequivocally scored as delayed-but-complete (“2”) or complete (“3”) perfusion by ASL, suggesting that DSA analysis may underestimate compensation in certain situations. Thus, the ASPECTS analysis supports higher confidence and less equivocality of ASL data, likely due to its volumetric, 3D nature. Given the importance of accurate localization for properly delineating regions of hypoperfusion (and, for example, computing tissue volumes), the ASL approach may in fact surpass DSA in this area.

Another powerful advantage of ASL over DSA is its unique ability to quantify microvascular $\mathrm{CBF}$ in absolute units (milliliters $/ 100 \mathrm{~g} / \mathrm{min}$ ), which can reveal subtle $\mathrm{CBF}$ changes not obvious qualitatively. An approach to measure CBF in ACA, MCA, and PCA territories is introduced in this study, with a vascular territory mask offering a standardized means to enable interterritorial and interhemispheric comparisons. ${ }^{16,17}$ Due to the known heterogeneity of normal CBF values in children of different ages, ${ }^{16,23}$ an arguably more useful quantitative metric of perfusion for group comparison is the $\mathrm{CBF} \mathrm{CV}$, which provides a measure of perfusion variability within a vascular territory, with lower values suggesting more homogeneous perfusion. PASL-CBF values in regions of transit delay demonstrate high variability (particularly when compared to asymptomatic siblings), despite more homogeneous perfusion seen on DSA and VSASL. This is presumably due to averaging of erroneously high CBF voxels (due to the arterial transit artifact) with erroneously low $\mathrm{CBF}$ voxels (due to the apparent perfusion deficit).

In contrast to PASL, VSASL-CBF CV values in regions affected by transit delay in patients were similar to those of normal regions in the asymptomatic siblings (Fig. 3), corroborating the homogeneous (but delayed) territorial parenchymal phase demonstrated by DSA. However, while the absolute difference in VSASL-CBF CV between patients and asymptomatic siblings was small (0.04), this difference was still statistically significant at $P<.005$, suggesting that despite generally preserved perfusion in 
these patients, there may be subtly increased variability due to the disease process/surgery itself. This may be interesting to investigate in future studies and suggests added value of VSASL over DSA alone. Notably, when using VSASL within a single individual (for example, to compare diseased with healthy regions or follow serially), accurate absolute CBF will be crucial.

Unlike in the patient group, no statistical difference was found between PASL and VSASL-CBF measurements in the asymptomatic siblings, supporting that both ASL methods give accurate results in the absence of significant stenoses and transit delays.

This study suggests that PASL and VSASL together provide a robust, noninvasive, cerebral hemodynamic assessment in childhood moyamoya, allowing evaluation of parenchymal perfusion by VSASL and regional transit delay via PASL. Cerebral perfusion is universally acknowledged as a critically important parameter related to clinical outcome $e^{24-28}$ and may even be more predictive of outcome than angiographic state. ${ }^{25}$ Such functional information may be used preoperatively to monitor progression and better inform future surgical needs, and postoperatively to measure the combined impact of surgical and native collaterals on regional $\mathrm{CBF}$ and also to monitor postsurgical progression. Given our findings, both PASL and VSASL have now become part of the moyamoya MR imaging protocol at our home institution of Boston Children's Hospital (a major moyamoya referral center) and are routinely performed on all patients with moyamoya for both initial assessment and periodic follow-up. VSASL has also been added to certain adult and pediatric protocols at a nearby institution (Massachusetts General Hospital), with preliminary results suggesting that VSASL-CBF may have greater predictive value relative to PASL in suspected ischemic stroke. ${ }^{29}$

Several limitations of this study must be mentioned. First, ASL is an inherently SNR-limited technique, necessitating large voxels and low resolution. Future studies will incorporate a background-suppressed, segmented $3 \mathrm{D}$ readout to improve SNR and resolution. PASL may also be replaced by pCASL, which is a higher SNR spatial-based ASL approach. An artifact unique to VSASL is CSF contamination, particularly within the basal cisterns/ventricles, due to velocity-based labeling. A CSF-suppressed approach is under development.

While bolus propagation is similar for both PASL and DSA, the PASL bolus travels at the velocity of inflowing arterial blood, whereas the DSA bolus may also be affected by the force/rate of injection. Combined with low DSA temporal resolution $(500 \mathrm{~ms})$, correspondence between DSA phase and PASL-PLD is an approximation and thus included in our qualitative assessment only.

One important limitation of our PASL/VSASL approach is that while it detects transit delay/collaterals and measures $\mathrm{CBF}$, it does not make a distinction between surgical or native collaterals. This could be potentially addressed using new vessel-encoded ASL techniques to image collateral pathways. ${ }^{30}$

Other spatial-based ASL approaches to mitigate arterial transit delay effects in moyamoya have been attempted. A recent study evaluated pCASL with both long PLDs ( $>4$ seconds) and multiPLDs (0.7-3 seconds) for moyamoya CBF measurement. ${ }^{31}$ This study found that even multi-PLD approaches underestimate CBF in the presence of long transit delays, whereas PLDs of $>4$ seconds result in stronger correlation with ${ }^{15} \mathrm{O}-\mathrm{PET} \mathrm{CBF}$. Such long delays, however, will result in marked signal loss due to T1 decay and may also violate a fundamental ASL assumption: that the magnetic label remains within the microvascular tree during the experiment. At PLDs of $>4$ seconds, labeled spins will start clearing into the venous circulation in regions without significant transit delay, leading to $\mathrm{CBF}$ underestimation. This feature becomes especially relevant in patients with heterogeneous and/or unilateral disease. VSASL, on the other hand, will be accurate in all regions, insensitive to large variations in transit delay across the brain.

Acetazolamide challenge to measure cerebral vascular reserve (typically with SPECT/PET ${ }^{32}$ and potentially $\mathrm{ASL}^{33}$ ) is performed in many moyamoya centers and can provide important information about hemodynamic status and postoperative clinical outcome. $^{32}$ Because this is not routinely performed at Boston Children's Hospital, we are unable to draw comparisons with cerebral vascular reserve metrics. We assert, however, that ASL methods presented here may complement cerebral vascular reserve and can be performed without administration of exogenous agents and on routine follow-up MRIs. Moreover, if cerebral vascular reserve measurement is desired using MR imaging, it should be more accurate using a VSASL-based approach since moyamoya transit delay will decrease accuracy in spatial-based ASL-cerebral vascular reserve approaches. ${ }^{34,35}$ Of note, VSASL will accurately measure $\mathrm{CBF}$ even in the acetazolamide-increased $\mathrm{CBF}$ state; while higher $\mathrm{CBF}$ will shift the leading edge of the magnetic label downstream toward the capillary bed, the $\mathrm{CBF}$ measurement will not be adversely affected. ${ }^{35,36}$

Finally, lack of a quantitative criterion standard for comparison/validation, such as ${ }^{15} \mathrm{O}$-PET or xenon-enhanced CT is another important limitation; these methods, however, require ionizing radiation and exogenous agents and are not generally feasible in children. We thus relied on DSA as a qualitative criterion standard because it was part of the clinical protocol at Boston Children's Hospital.

\section{CONCLUSIONS}

We present a combined VSASL and PASL approach for imaging perfusion and transit delay, respectively, in pediatric moyamoya, using the temporal and spatial vascular patterns on DSA as the criterion standard comparison. Due to its insensitivity to arterial transit delays, VSASL offers a powerful new way to accurately image perfusion in moyamoya and provides important spatial and quantitative $\mathrm{CBF}$ information unobtainable by DSA. Such an approach may further characterize disease progression and response to therapy. Additional studies to assess this potential by comparing pre- and postoperative data are necessary and underway.

Disclosures: Divya S. Bolar-UNRELATED: Employment: Massachusetts General Hospital, Comments: I was a resident, then fellow, in the Radiology Department at Massachusetts General Hospital from 2013 to 2018 and was paid as such; Grants/Grants Pending: Radiological Society of North America Resident Grant, Comments: I received a Radiological Society of North America Resident Grant in 2016; these funds were used for other related projects and not directly for this project (which started before I received the grant). Elfar AdalsteinssonUNRELATED: Grants/Grants Pending: National Institutes of Health, Comments: National Institutes of Health 1U01HD087211-01 and 5R01EB017337-02, National Institutes of Health applications under review*; Royalties: Stanford Licensing Office, Comments: patents on MRI methods issued by Stanford University. Borjan Gagoski-UNRELATED: Grants/Grants Pending: National Institutes of Health 1U01HD087211-01 and 5R01EB017337-02.* Bruce R. Rosen—UNRELATED: Grants/Grants 
Pending: National Institutes of Health P41-EB015896.* P. Ellen Grant-UNRELATED: Grants/Grants Pending: National Institutes of Health 1U01HD087211-01 and 5R01EB017337-02.* *Money paid to the institution.

\section{REFERENCES}

1. Lin N, Smith ER, Scott RM, et al. Safety of neuroangiography and embolization in children: complication analysis of 697 consecutive procedures in 394 patients. J Neurosurg Pediatr 2015;16:43238 CrossRef Medline

2. Robertson RL, Chavali RV, Robson CD, et al. Neurologic complications of cerebral angiography in childhood moyamoya syndrome. Pediatr Radiol 1998;28:824-29 CrossRef Medline

3. Calamante F, Ganesan V, Kirkham FJ, et al. MR perfusion imaging in moyamoya syndrome: potential implications for clinical evaluation of occlusive cerebrovascular disease. Stroke 2001;32:2810-16 CrossRef Medline

4. Zaharchuk G, Do HM, Marks MP, et al. Arterial spin-labeling MRI can identify the presence and intensity of collateral perfusion in patients with moyamoya. Stroke 2011;42:2485-91 CrossRef Medline

5. Wong EC, Cronin M, Wu WC, et al. Velocity-selective arterial spin labeling. Magn Reson Med 2006;55:1334-41 Medline

6. Qiu D, Straka M, Zun Z, et al. CBF measurements using multidelay pseudocontinuous and velocity-selective arterial spin labeling in patients with long arterial transit delays: comparison with xenon CT CBF. J Magn Reson Imaging 2012;36:110-19 CrossRef Medline

7. US Food and Drug Administration. Xenon Xe 133 Gas. Washington, DC: US Food and Drug Administration; 2016 https://www. accessdata.fda.gov/drugsatfda_docs/label/2016/018327s005lbl.pdf. Accessed December 26, 2016

8. Wu W, Wong E. Intravascular effect in velocity-selective arterial spin labeling. Conf Proc IEEE Eng Med Biol Soc 2005;6:5790-93 Medline

9. Alsop DC, Detre JA, Golay X, et al. Recommended implementation of arterial spin-labeled perfusion MRI for clinical applications: a consensus of the ISMRM perfusion study group and the European consortium for ASL in dementia. Magn Reson Med 2015;73:102-16 CrossRef Medline

10. Buxton RB, Frank LR, Wong EC, et al. A general kinetic model for quantitative perfusion imaging with arterial spin labeling. Magn Reson Med 1998;40:383-96 CrossRef Medline

11. Cavusoglu M, Pfeuffer J, Uğurbil K, et al. Comparison of pulsed arterial spin labeling encoding schemes and absolute perfusion quantification. Magn Reson Imaging 2009;27:1039-45 CrossRef Medline

12. Kim JJ, Fischbein NJ, Lu Y, et al. Regional angiographic grading system for collateral flow: correlation with cerebral infarction in patients with middle cerebral artery occlusion. Stroke 2004; 35:1340-44 CrossRef Medline

13. Fonov V, Evans AC, Botteron $\mathrm{K}$, et al. Unbiased average ageappropriate atlases for pediatric studies. Neuroimage 2011;54: 313-27 CrossRef Medline

14. Mutsaerts HJ, van Dalen JW, Heijtel DF, et al. Cerebral perfusion measurements in elderly with hypertension using arterial spin labeling. PLoS One 2015;10:e0133717 CrossRef Medline

15. Tatu L, Moulin T, Bogousslavsky J, et al. Arterial territories of the human brain: cerebral hemispheres. Neurology 1998;50:1699-1708 CrossRef Medline

16. Biagi L, Abbruzzese A, Bianchi MC, et al. Age dependence of cerebral perfusion assessed by magnetic resonance continuous arterial spin labeling. J Magn Reson Imaging 2007;25:696-702 CrossRef Medline

17. Wang J, Licht DJ. Pediatric perfusion MR imaging using arterial spin labeling. Neuroimaging Clin N Am 2006;16:149-67, ix CrossRef Medline

18. Detre JA, Samuels OB, Alsop DC, et al. Noninvasive magnetic resonance imaging evaluation of cerebral blood flow with acetazolamide challenge in patients with cerebrovascular stenosis. J Magn Reson Imaging 1999;10:870-75 CrossRef Medline

19. Zaharchuk G. Arterial spin label imaging of acute ischemic stroke and transient ischemic attack. Neuroimaging Clin N Am 2011; 21:285-301, x CrossRef Medline
20. Deibler AR, Pollock JM, Kraft RA, et al. Arterial spin-labeling in routine clinical practice, part 1: technique and artifacts. AJNR Am J Neuroradiol 2008;29:1228-34 CrossRef Medline

21. Mutke MA, Madai VI, von Samson-Himmelstjerna FC, et al. Clinical evaluation of an arterial-spin-labeling product sequence in steno-occlusive disease of the brain. PLoS One 2014;9:e87143 CrossRef Medline

22. Schmid S, Heijtel DF, Mutsaerts HJ, et al. Comparison of velocityand acceleration-selective arterial spin labeling with $[15 \mathrm{O}] \mathrm{H} 2 \mathrm{O}$ positron emission tomography. J Cereb Blood Flow Metab 2015; 35:1296-1303 CrossRef Medline

23. Wang J, Licht DJ, Jahng G-H, et al. Pediatric perfusion imaging using pulsed arterial spin labeling. J Magn Reson Imaging 2003; 18:404-13 CrossRef Medline

24. Ishii R, Takeuchi S, Ibayashi $\mathrm{K}$, et al. Intelligence in children with moyamoya: evaluation after surgical treatments with special reference to changes in cerebral blood flow. Stroke 1984; 15:873-77 CrossRef Medline

25. McAuley DJ, Poskitt K, Steinbok P. Predicting stroke risk in pediatric moyamoya with xenon-enhanced computed tomography. Neurosurgery 2004;55:327-32; discussion 332-33 CrossRef Medline

26. Touho H, Karasawa J, Ohnishi H. Preoperative and postoperative evaluation of cerebral perfusion and vasodilatory capacity with 99mTc-HMPAO SPECT and acetazolamide in childhood moyamoya. Stroke 1996;27:282-289 CrossRef Medline

27. Lee M, Zaharchuk G, Guzman R, et al. Quantitative hemodynamic studies in moyamoya: a review. Neurosurg Focus 2009;26:E5 CrossRef Medline

28. Ikezaki K, Matsushima T, Kuwabara Y, et al. Cerebral circulation and oxygen metabolism in childhood moyamoya: a perioperative positron emission tomography study. J Neurosurg 1994; 81:843-50 CrossRef Medline

29. Bolar DS, Rosen BR, Schaefer PW. Comparison of Velocity-selective and Pulsed ASL Perfusion MRI in Patients with Suspected Cerebral Cortical Ischemia. In: Proceedings of the Annual Meeting of American Society of Neuroradiology, Vancouver, BC. June 6, 2018:1-4

30. Wu B, Wang X, Guo J, et al. Collateral circulation imaging: MR perfusion territory arterial spin-labeling at 3T. AJNR Am J Neuroradiol 2008;29:1855-60 CrossRef Medline

31. Fan AP, Guo J, Khalighi MM, et al. Long-delay arterial spin labeling provides more accurate cerebral blood flow measurements in moyamoya patients: a simultaneous positron emission tomography/MRI study. Stroke 2017;48:2441-49 CrossRef Medline

32. So Y, Lee H-Y, Kim SK, et al. Prediction of the clinical outcome of pediatric moyamoya with postoperative basal/acetazolamide stress brain perfusion SPECT after revascularization surgery. Stroke 2005;36:1485-89 CrossRef Medline

33. Yun TJ, Paeng JC, Sohn C-H, et al. Monitoring cerebrovascular reactivity through the use of arterial spin labeling in patients with moyamoya. Radiology 2016;278:205-13 CrossRef Medline

34. Blauwblomme T, Lemaitre H, Naggara O, et al. Cerebral blood flow improvement after indirect revascularization for pediatric moyamoya: a statistical analysis of arterial spin-labeling MRI. AJNR Am J Neuroradiol 2016;37:706-12 CrossRef Medline

35. Wu WC, Wong EC. Feasibility of velocity selective arterial spin labeling in functional MRI. J Cereb Blood Flow Metab 2007;27:83138 CrossRef Medline

36. Wu WC, Wong EC. Intravascular effect in velocity-selective arterial spin labeling: the choice of inflow time and cutoff velocity. Neuroimage 2006;32:122-28 CrossRef Medline

37. Suzuki J, Takaku A. Cerebrovascular "moyamoya" disease: disease showing abnormal net-like vessels in base of brain. Arch Neurol 1969;20:288-99 CrossRef Medline

38. Matsushima T, Inoue T, Suzuki SO, et al. Surgical treatment of moyamoya disease in pediatric patients: comparison between the results of indirect and direct revascularization procedures. Neurosurgery 1992;31:401-05 CrossRef Medline 